\title{
ON THE DISTRIBUTION AND $q$-VARIATION OF THE SOLUTION TO THE HEAT EQUATION WITH FRACTIONAL LAPLACIAN
}

BY

\author{
Z. MAHDI KHALIL (Lille) AND C. A. TUDOR* (Lille)
}

Abstract. We study the probability distribution of the solution to the linear stochastic heat equation with fractional Laplacian and white noise in time and white or correlated noise in space. As an application, we deduce the behavior of the $q$-variations of the solution in time and in space.

2010 AMS Mathematics Subject Classification: Primary: 60G15; Secondary: 60H05, 60G18.

Key words and phrases: Stochastic heat equation, fractional Brownian motion, fractional Laplacian, quadratic variation.

\section{INTRODUCTION}

In this work we study the probability distribution and other properties of the solution to the fractional stochastic heat equation driven by an additive Gaussian noise which is white in time and white or correlated in space. Here, the word "fractional" concerns the appearance of the fractional Laplacian operator in the equation and it does not refer to the noise.

Fractional stochastic (partial) differential equations are used to model various phenomena in a large number of scientific branches, including statistical mechanics, theoretical physics, hydrology or economics. For instance, the (deterministic) fractional heat equation (i.e. the deterministic counterpart of (B.1) and (4.1) below) describes the heat propagation in inhomogeneous media being related to anomalous diffusion (see e.g. [17] ], [5]). Their stochastic counterparts (3.J) and (4.J) are valuable models for similar phenomena with random effects, including random effects with thermal memory (see [5], [16], [17]]). The link between the fractional (stochastic) differential equations and the conservation law has been studied in [1]] or [22] among many others. See also the monograph [13] for an extensive list of references.

* The author acknowledges support from LABEX CEMPI (ANR-11-LABX-0007-01) and MATHAMSUD PROJECT SARC (19-MATH-06). 
Several recent and less recent papers showed an interesting connection between the solution to the classical stochastic linear heat equation and some stochastic processes related to the (bi)fractional Brownian motion. Consider the stochastic partial differential equation

$$
\frac{\partial}{\partial t} u(t, x)=\Delta u(t, x)+\dot{W}(t, x), \quad t \geqslant 0, x \in \mathbb{R}^{d},
$$

with vanishing initial condition $u(0, x)=0$ for every $x \in \mathbb{R}^{d}$. In (ㅍ.d), we denoted by $\Delta$ the standard Laplacian on $\mathbb{R}^{d}$ and by $W$ the random noise which is defined as a centered Gaussian process $\left(W(t, A), t \geqslant 0, A \in \mathcal{B}_{b}\left(\mathbb{R}^{d}\right)\right)$ with covariance

$$
\mathbf{E} W(t, A) W(s, B)=(t \wedge s) \int_{A} \int_{B}\|x-y\|^{-\gamma} d x d y \quad \text { if } \gamma \in(0, d)
$$

and

$$
\mathbf{E} W(t, A) W(s, B)=(t \wedge s) \lambda(A \cap B) \quad \text { if } \gamma=0 .
$$

We denoted by $\|\cdot\|$ the Euclidean norm in $\mathbb{R}^{d}$ and by $\mathcal{B}_{b}\left(\mathbb{R}^{d}\right)$ the class of bounded Borel sets in $\mathbb{R}^{d}$.

In the first case, the noise is said to be white in time and correlated in space with spatial correlation given by the Riesz kernel. In the second case, we have a time-space white noise, i.e. the noise behaves as a Wiener process both in time and in space.

The solution to $(\mathbb{L}$. $\mathbb{C}$ ) is usually defined in the mild sense, i.e. as the Wiener integral with respect to the noise $W$, by

$$
u(t, x)=\int_{0}^{t} \int_{\mathbb{R}^{d}} G(t-s, x-y) W(d s, d y),
$$

where $G$ is the fundamental solution of the heat equation, i.e. the deterministic function which solves $\frac{\partial}{\partial t} u(t, x)=\Delta u(t, x)$.

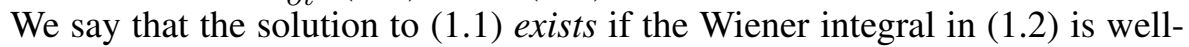
defined in $L^{2}(\Omega)$. We know (see e.g. [23]) that the necessary and sufficient condition for the existence of the solution is

$$
d<2+\gamma,
$$

which means $d=1$ in the case of the time-space white noise $(\gamma=0)$.

Also, the solution is connected to the bifractional Brownian motion. Recall that (see [9]), given constants $H \in(0,1)$ and $K \in(0,1]$, the bifractional Brownian motion (bi-fBm for short) $\left(B_{t}^{H, K}\right)_{t \in[0, T]}$ is a centered Gaussian process with covariance

$$
R^{H, K}(t, s):=R(t, s)=\frac{1}{2^{K}}\left(\left(t^{2 H}+s^{2 H}\right)^{K}-|t-s|^{2 H K}\right), \quad s, t \in[0, T] .
$$


In particular, for $K=1, B^{H, 1}:=B^{H}$ is the fractional Brownian motion with Hurst parameter $H \in(0,1)$.

It is known that for every fixed $x \in \mathbb{R}^{d}$, the process $(u(t, x), t \geqslant 0)$ given by (L.2) coincides in distribution, modulo a constant, with the bifractional Brownian motion with parameters $H=\frac{1}{2}$ and $K=1-(d-\gamma) / 2$ (so $H=K=\frac{1}{2}$ if $\gamma=0$ and $d=1$ ).

Concerning the behavior with respect to the space variable, we know from [4] that for fixed $t>0$ and for $d=1$, the process $(u(t, x), x \in \mathbb{R})$ has the same law as a Brownian motion plus an independent Gaussian process with $C^{\infty}$ sample paths.

When the noise is correlated in time, for example when the noise behaves as a fractional Brownian motion with respect to the time variable, there are also links between the law of the solution to the heat equation and the fractional processes, see e.g. [7] or [24].

All these connections are very useful to deduce various properties of the solution to the heat equation by using known results for the fractional Brownian motion.

In this work, our purpose is to do a similar analysis for the solution to the fractional stochastic heat equation, i.e. when the Laplacian is replaced by the fractional Laplacian of order $\alpha \in(1,2]$, denoted by $-(-\Delta)^{\alpha / 2}$, in equation (IL.I). We want to understand the influence of the parameter $\alpha$ on the law and on the sample paths regularity of the solution. We give a necessary and sufficient condition for the existence of the solution for the fractional heat equation and study the connection with fractional Brownian motion and related processes. We prove the following results: while with respect to the time variable the solution still remains a bifractional Brownian motion (whose Hurst parameters will be explicitly given), the behavior of the solution in space will be related to the isotropic fractional Brownian sheet. The result was known for dimension $d=1$ from [4], but for dimension $d \geqslant 2$, we notice the appearance, for the first time in the literature, of the multiparameter fractional Brownian motion of isotropic type.

We apply these findings to study the $q$-variations of the solution to the fractional heat equation. For a stochastic process $\left(X_{t}\right)_{t \geqslant 0}$, we will consider two types of variations:

- the exact $q$-variations $(q \in(0, \infty))$ over an interval $\left[A_{1}, A_{2}\right]$, meaning the limit in probability of the sequence

$$
V^{q, n}(X)=\sum_{i=0}^{n-1}\left|X_{t_{i+1}}-X_{t_{i}}\right|^{q}
$$

if $t_{i}=A_{1}+\frac{i}{n}\left(A_{2}-A_{1}\right), i=0, \ldots, n$, constitutes a partition of $\left[A_{1}, A_{2}\right]$;

- the renormalized $q$-variations (which are usually defined for self-similar stochastic processes and for $q \geqslant 1$ integer) as the limit in distribution, as $n \rightarrow \infty$, 
of the sequence

$$
V_{q, n}(X)=\sum_{i=0}^{n-1}\left[\frac{\left(X_{t_{i+1}}-X_{t_{i}}\right)^{q}}{\mathbf{E}\left(X_{t_{i+1}}-X_{t_{i}}\right)^{q}}-\mu_{q}\right],
$$

where $\mu_{q}=\mathbf{E} Z^{q}$ with $Z \sim N(0,1)$ (we will use this notation throughout the paper).

More details on these notions will be given below in Section 2. In the same Section 2, we also prove some new results concerning the variations of the perturbed fractional Brownian motion. In Section 3 we study the solution to the fractional heat equation with time-space white noise while Section 4 is devoted to the analysis of the white-colored noise case.

\section{VARIATIONS OF THE PERTURBED FRACTIONAL BROWNIAN MOTION}

In this section we introduce the notion of exact $q$-variation and of renormalized $q$-variation for stochastic processes. We will also recall some known results for the fractional Brownian motion and bifractional Brownian motion. In the last part, we obtain the $q$-variation for a perturbed $\mathrm{fBm}$, i.e. the sum of an $\mathrm{fBm}$ and of a smooth process. This result for the perturbed $\mathrm{fBm}$ will be applied several times in this work.

2.1. Exact and renormalized $q$-variations for stochastic processes. We first define the concept of exact $q$-variation for stochastic processes.

Definition 2.1. Let $A_{1}<A_{2}$ and, for $n \geqslant 1$, let $t_{i}=A_{1}+\frac{i}{n}\left(A_{2}-A_{1}\right)$ for $i=0, \ldots, n$. A continuous stochastic process $\left(X_{t}\right)_{t \geqslant 0}$ admits a $q$-variation (or a variation of order $q$ ) over the interval $\left[A_{1}, A_{2}\right]$ if the sequence

$$
V_{\left[A_{1}, A_{2}\right]}^{n, q}(X)=\sum_{i=0}^{n-1}\left|X_{t_{i+1}}-X_{t_{i}}\right|^{q}
$$

converges in probability as $n \rightarrow \infty$. The limit, when it exists, is called the exact $q$-variation of $X$ over the interval $\left[A_{1}, A_{2}\right]$.

If $\left[A_{1}, A_{2}\right]=[0, t]$, we will simply write $V_{\left[A_{1}, A_{2}\right]}^{n, q}(X):=V_{t}^{n, q}(X)$. Moreover, if $t=1$, we write $V_{t}^{n, q}(X):=V^{q, n}$. In the case $q=2$ the limit of $V^{2, n}$ is called the quadratic variation, while for $q=3$ we have the cubic variation.

Let us recall the following result concerning the $q$-variation of the bifractional Brownian motion (see Proposition 1 in [14]).

Proposition 2.1. If $\left(B_{t}^{H, K}\right)_{t \geqslant 0}$ is a bi-fBm with Hurst parameters $H \in$ $(0,1), K \in(0,1]$, then $B^{H, K}$ admits a variation of order $1 /(H K)$ over any interval $\left[A_{1}, A_{2}\right]$, which is equal to $C_{H, K}\left(A_{2}-A_{1}\right)$, where $Z$ is a standard normal 
random variable and

$$
C_{H, K}=2^{(1-K) / 2} \mathbf{E}|Z|^{1 /(H K)} .
$$

By taking $K=1$, we notice that the fractional Brownian motion has $1 / H$ variation over the interval $\left[A_{1}, A_{2}\right]$ given by $\mathbf{E}|Z|^{1 / H}\left(A_{2}-A_{1}\right)$.

We will also study the asymptotic behavior of the normalized $q$-variation of the solution to the fractional heat equation. Even if this notion is usually studied for self-similar stochastic processes, one can discuss it for more general stochastic processes. To define this object, let us recall the case of the fractional Brownian motion. Let $B^{H}$ be an $\mathrm{fBm}$ with Hurst parameter $H \in(0,1)$ and define

$$
V_{q, n}\left(B^{H}\right):=\sum_{i=0}^{n-1}\left[\frac{n^{H q}}{\left(A_{2}-A_{1}\right)^{H q}}\left(B_{t_{i+1}}^{H}-B_{t_{i}}^{H}\right)^{q}-\mu_{q}\right] .
$$

This is called the (centered) renormalized q-variation because the random variable $\left(B_{t_{i+1}}^{H}-B_{t_{i}}^{H}\right)^{q}$ is normalized, i.e. the expectation of

$$
\frac{n^{H q}}{\left(A_{2}-A_{1}\right)^{H q}}\left(B_{t_{i+1}}^{H}-B_{t_{i}}^{H}\right)^{q}
$$

is $\mu^{q}=\mathbf{E}|Z|^{q}$. Many papers treated recently the limit behavior in distribution of the renormalized $q$-variations for various stochastic processes, see e.g. [23] and the references therein.

We recall the following result concerning the variations of the fractional Brownian motion (see [2], [3], [6]; the reader may also consult Section 1 in [18] for a survey of these results). We will restrict ourselves below to the case when the Hurst parameter $H$ is less than or equal to $\frac{1}{2}$ since only this case will be needed in the sequel. That is, if $q \geqslant 2$ is an integer and $H \in\left(0, \frac{1}{2}\right]$, then

$$
\frac{1}{\sqrt{n}} V_{q, n}(B):=\frac{1}{\sqrt{n}} \sum_{i=0}^{n-1}\left[\frac{n^{H q}}{\left(A_{2}-A_{1}\right)^{H q}}\left(B_{t_{i+1}}-B_{t_{i}}\right)^{q}-\mu_{q}\right] \rightarrow N\left(0, \sigma_{H, q}^{2}\right),
$$

where $\sigma_{H, q}^{2}$ denotes a strictly positive constant depending on $q$ and $H$.

We will analyze the asymptotic behavior of the renormalized $q$-variation for various stochastic processes, not necessarily self-similar. For a general process $\left(X_{t}\right)_{t \geqslant 0}$, by studying the asymptotic behavior of the renormalized $q$-variation we will generally mean to find a constant $\mu \in \mathbb{R}$ and two deterministic sequences $f(n)$ and $g(n)$ which converge to zero as $n \rightarrow \infty$ such that $\mathbf{E}\left[f(n)^{-1}\left(X_{t_{i+1}}-X_{t_{i}}\right)\right]^{2}$ is close to one and

$$
g(n) \sum_{i=0}^{n-1}\left[\frac{\left(X_{t_{i+1}}-X_{t_{i}}\right)^{q}}{f(n)}-\mu_{q}\right]
$$

converges in distribution to a non-trivial limit as $n \rightarrow \infty$. We illustrate below the case of the perturbed $\mathrm{fBm}$. 
2.2. $q$-variation of the perturbed fractional Brownian motion. In the next sections, we will see that the solution to the fractional heat equation can be decomposed as the sum of an $\mathrm{fBm}$ (with Hurst parameter less than or equal to one half) and a smooth process. Therefore, we need to understand the variations of such stochastic processes. This can be relatively easily obtained from the results known for the $\mathrm{fBm}$ and recalled above.

Concerning the asymptotic behavior of the exact and renormalized $q$-variation of the sum of an $\mathrm{fBm}$ and a smooth process, we have the following lemma.

LEMMA 2.1. Let $\left(B_{t}^{H}\right)_{t \geqslant 0}$ be an $\mathrm{fBm}$ with $H \in\left(0, \frac{1}{2}\right]$ and consider a centered Gaussian process $\left(X_{t}\right)_{t \geqslant 0}$ such that

$$
\mathbf{E}\left|X_{t}-X_{s}\right|^{2} \leqslant C|t-s|^{2} \quad \text { for every } s, t \geqslant 0 .
$$

Define

$$
Y_{t}^{H}=B_{t}^{H}+X_{t} \quad \text { for every } t \geqslant 0 .
$$

Then:

(1) The process $Y$ has $1 / H$-variation over the interval $\left[A_{1}, A_{2}\right]$ which is equal to

$$
\mathbf{E}|Z|^{1 / H}\left(A_{2}-A_{1}\right) .
$$

(2) Let

$$
V_{q, n}\left(Y^{H}\right):=\sum_{i=0}^{n-1}\left[\frac{n^{H q}}{\left(A_{2}-A_{1}\right)^{q H}}\left(Y_{t_{i+1}}^{H}-Y_{t_{i}}^{H}\right)^{q}-\mu_{q}\right] .
$$

Then, if $H \in\left(0, \frac{1}{2}\right)$ and $q \geqslant 2$, we have

$$
\frac{1}{\sqrt{n}} V_{q, n}\left(Y^{H}\right)=\frac{1}{\sqrt{n}} \sum_{i=0}^{n-1}\left[\frac{n^{H q}}{\left(A_{2}-A_{1}\right)^{q H}}\left(Y_{t_{i+1}}^{H}-Y_{t_{i}}^{H}\right)^{q}-\mu_{q}\right] \rightarrow N\left(0, \sigma_{H, q}^{2}\right) .
$$

If $H=\frac{1}{2}, q=2$ and the process $\left(X_{t}\right)_{t \geqslant 0}$ is adapted to the filtration generated by $B$, then

$$
\frac{1}{\sqrt{n}} V_{2, n}\left(Y^{H}\right)=\frac{1}{\sqrt{n}} \sum_{i=0}^{n-1}\left[\frac{n^{2 H}}{\left(A_{2}-A_{1}\right)^{2 H}}\left(Y_{t_{i+1}}^{H}-Y_{t_{i}}^{H}\right)^{2}-1\right] \rightarrow N\left(0, \sigma_{1 / 2,2}^{2}\right) .
$$

Pr o of. To prove (1), we use the Minkowski inequality to write

$$
\begin{aligned}
\left(\sum_{i=0}^{n-1} \mid B_{t_{i+1}}^{H}\right. & \left.-\left.B_{t_{i}}^{H}\right|^{1 / H}\right)^{H}-\left(\sum_{i=0}^{n-1}\left|X_{t_{i+1}}-X_{t_{i}}\right|^{1 / H}\right)^{H} \\
& \leqslant\left(\sum_{i=0}^{n-1}\left|Y_{t_{i+1}}^{H}-Y_{t_{i}}^{H}\right|^{1 / H}\right)^{H} \\
& \leqslant\left(\sum_{i=0}^{n-1}\left|B_{t_{i+1}}^{H}-B_{t_{i}}^{H}\right|^{1 / H}\right)^{H}+\left(\sum_{i=0}^{n-1}\left|X_{t_{i+1}}-X_{t_{i}}\right|^{1 / H}\right)^{H}
\end{aligned}
$$


Since, by Proposition 2.11, the sequence

$$
\sum_{i=0}^{n-1}\left|B_{t_{i+1}}^{H}-B_{t_{i}}^{H}\right|^{1 / H}
$$

converges in probability, as $n \rightarrow \infty$, to the desired limit $\mathbf{E}|Z|^{1 / H}\left(A_{2}-A_{1}\right)$, it suffices to show that $\sum_{i=0}^{n-1}\left|X_{t_{i+1}}-X_{t_{i}}\right|^{1 / H}$ converges to zero. We have, via ([2.3) and Hölder's inequality, as $n \rightarrow \infty$,

$$
\mathbf{E} \sum_{i=0}^{n-1}\left|X_{t_{i+1}}-X_{t_{i}}\right|^{1 / H} \leqslant \sum_{i=0}^{n-1} \mathbf{E}\left(\left|X_{t_{i+1}}-X_{t_{i}}\right|^{2}\right)^{1 /(2 H)} \leqslant n^{1-1 / H} \rightarrow 0 .
$$

Let us prove (2). Consider first the situation $H \in\left(0, \frac{1}{2}\right)$. By using Newton's formula we can write

$$
V_{q, n}\left(Y^{H}\right)=V_{q, n}(B)+R_{n}
$$

where

$$
R_{n}=\frac{n^{H q}}{\sqrt{n}} \sum_{r=0}^{q-1} C_{q}^{r} \sum_{i=0}^{n-1}\left(B_{t_{i+1}}-B_{t_{i}}\right)^{r}\left(X_{t_{i+1}}-X_{t_{i}}\right)^{q-r}:=\sum_{r=0}^{q-1} R_{n, r} .
$$

Now, it suffices to show that $\frac{1}{\sqrt{n}} R_{n, r}$ converges to zero in $L^{1}(\Omega)$ for every $r=$ $0, \ldots, q-1$. Using ([2.3), we have for every $s, t \geqslant 0$ and for $r=0, \ldots, q-1$

$$
\mathbf{E}\left|X_{t}-X_{s}\right|^{2(q-r)} \leqslant C|t-s|^{2(q-r)}
$$

and then we can write, for $r=0, \ldots, q-1$,

$$
\begin{aligned}
\mathbf{E} \frac{1}{\sqrt{n}}\left|R_{n, r}\right| & \leqslant \frac{1}{\sqrt{n}} \sum_{i=0}^{n-1}\left(\mathbf{E}\left(B_{t_{i+1}}-B_{t_{i}}\right)^{2 r}\right)^{1 / 2}\left(\mathbf{E}\left(X_{t_{i+1}}-X_{t_{i}}\right)^{2(q-r)}\right)^{1 / 2} \\
& \leqslant C n^{(H-1)(q-r)+1 / 2} \leqslant C n^{H-1 / 2}
\end{aligned}
$$

and this converges to zero as $n \rightarrow \infty$ since $H<\frac{1}{2}$.

If $H=\frac{1}{2}$ and $q=2$, we have

$$
\begin{aligned}
\frac{1}{\sqrt{n}} V_{2, n}\left(Y^{1 / 2}\right)= & \frac{1}{\sqrt{n}} V_{2, n}(B)+\frac{2}{\sqrt{n}} \sum_{i=0}^{n-1} \frac{n}{A_{2}-A_{1}}\left(B_{t_{i+1}}-B_{t_{i}}\right)\left(X_{t_{i+1}}-X_{t_{i}}\right) \\
& +\frac{1}{\sqrt{n}} \frac{n}{A_{2}-A_{1}} \sum_{i=0}^{n-1}\left(X_{t_{i+1}}-X_{t_{i}}\right)^{2} .
\end{aligned}
$$

Clearly, by (2.3)), we have, as $n \rightarrow \infty$,

$$
\sqrt{n} \mathbf{E} \sum_{i=0}^{n-1}\left(X_{t_{i+1}}-X_{t_{i}}\right)^{2} \leqslant c n^{-1 / 2} \rightarrow 0,
$$


and since $B$ is a martingale and $X$ is adapted to the filtration of $B$, we get, as $n \rightarrow \infty$,

$$
\begin{aligned}
& \mathbf{E}\left(\sqrt{n} \sum_{i=0}^{n-1}\left(B_{t_{i+1}}-B_{t_{i}}\right)\left(X_{t_{i+1}}-X_{t_{i}}\right)\right)^{2}=n \mathbf{E} \sum_{i=0}^{n-1}\left(B_{t_{i+1}}-B_{t_{i}}\right)^{2}\left(X_{t_{i+1}}-X_{t_{i}}\right)^{2} \\
& \leqslant n \sum_{i=0}^{n-1}\left(\mathbf{E}\left(B_{t_{i+1}}-B_{t_{i}}\right)^{4}\right)^{1 / 2}\left(\mathbf{E}\left(X_{t_{i+1}}-X_{t_{i}}\right)^{4}\right)^{1 / 2} \leqslant C n \sum_{i=0}^{n-1} \frac{1}{n} \frac{1}{n^{2}} \leqslant C \frac{1}{n} \rightarrow 0,
\end{aligned}
$$

which completes the proof.

Notice that when $H=\frac{1}{2}$ we need to assume that the process $X$ is adapted in order to apply the martingale property.

\section{HEAT EQUATION WITH FRACTIONAL LAPLACIAN DRIVEN BY TIME-SPACE WHITE NOISE}

We first consider the fractional heat equation driven by a time-space white noise. We study the existence, the probability distribution and the variations of the solution both in time and in space.

3.1. The equation and its solution. Consider the stochastic partial differential equation

$$
\frac{\partial}{\partial t} u(t, x)=-(-\Delta)^{\alpha / 2} u(t, x)+\dot{W}(t, x), \quad t \geqslant 0, x \in \mathbb{R}^{d},
$$

with vanishing initial condition $u(0, x)=0$ for every $x \in \mathbb{R}^{d}$. In (B.]), $-(-\Delta)^{\alpha / 2}$ denotes the fractional Laplacian with exponent $\alpha / 2, \alpha \in(1,2]$, and $W$ is a timespace white noise, i.e. $\left(W(t, A), t \geqslant 0, A \in \mathcal{B}\left(\mathbb{R}^{d}\right)\right)$ is a centered Gaussian field with covariance

$$
\mathbf{E} W(t, A) W(s, B)=(t \wedge s) \lambda(A \cap B),
$$

where $\lambda$ denotes the Lebesgue measure on $\mathbb{R}^{d}$. We refer to [10]-[12] for the precise definition and other properties of the fractional Laplacian operator. We will use here only the expression of the Green kernel $G_{\alpha}$ (or the fundamental solution) associated with the fractional Laplacian, i.e. the deterministic kernel that solves the heat equation without noise $\frac{\partial}{\partial t} u(t, x)=-(-\Delta)^{\alpha / 2} u(t, x)$. This Green kernel is defined through its Fourier transform

$$
\mathcal{F} G_{\alpha}(t, \cdot)(\xi)=e^{-t\|\xi\|^{\alpha}}, \quad t>0, \xi \in \mathbb{R}^{d},
$$

where $\mathcal{F} G_{\alpha}(t, \cdot)$ is the Fourier transform of the function $y \rightarrow G_{\alpha}(t, y)$.

The mild solution to (B.d) is understood in the mild sense, i.e.

$$
u(t, x)=\int_{0}^{t} \int_{\mathbb{R}^{d}} G_{\alpha}(t-u, x-z) W(d u, d z),
$$


where the integral $W(d u, d z)$ is a Wiener integral with respect to the Gaussian noise $W$.

First, we notice that the solution exists only in spatial dimension $d=1$.

Proposition 3.1. Let $\left(u(t, x), t \geqslant 0, x \in \mathbb{R}^{d}\right)$ be given by (3.3). Then the solution is well-defined if and only if $d=1$. Moreover, in this case, for every $T>0$

$$
\sup _{t \in[0, T], x \in \mathbb{R}} \mathbf{E}|u(t, x)|^{2}<\infty .
$$

Pro of. By the Wiener isometry, the Plancherel identity and the expression of the Fourier transform (3.2), we have for every $t>0, x \in \mathbb{R}^{d}$

$$
\begin{aligned}
\mathbf{E} u(t, x)^{2} & =\int_{0}^{t} d u \int_{\mathbb{R}^{d}} d z\left|G_{\alpha}(t-u, x-z)\right|^{2} \\
& =(2 \pi)^{-d} \int_{0}^{t} d u \int_{\mathbb{R}^{d}} d \xi\left|\mathcal{F} G_{\alpha}(u, \cdot)(\xi)\right|^{2} \\
& =(2 \pi)^{-d} \int_{0}^{t} d u \int_{\mathbb{R}^{d}} d \xi e^{-2 u\|\xi\|^{\alpha}}=C_{d, \alpha} \int_{0}^{t} d u u^{-d / \alpha}
\end{aligned}
$$

with $C_{d, \alpha}=(2 \pi)^{-d} \int_{\mathbb{R}^{d}} d \xi e^{-2\|\xi\|^{\alpha}}<\infty$. The integral $\int_{0}^{t} u^{-d / \alpha} d u$ is finite if and only if $1-d / \alpha>0$, which means $d<\alpha$ or $d=1$ since $\alpha \in(1,2]$. Moreover, for every $t \in[0, T], x \in \mathbb{R}^{d}$,

$$
\mathbf{E} u(t, x)^{2}=C_{1, \alpha} \frac{1}{1-1 / \alpha} T^{1-1 / \alpha}<\infty .
$$

Next, we will focus on the probability distribution of the solution in spatial dimension $d=1$. We will treat separately the behavior in time and in space.

3.2. Behavior in time. Let us consider here the process $(u(t, x), t \geqslant 0)$ with $x \in \mathbb{R}$ fixed. The distribution and the properties of this Gaussian process will follow easily from the computation of its covariance.

Proposition 3.2. For every $s, t \geqslant 0$ and $x \in \mathbb{R}$ we have

$$
\mathbf{E} u(t, x) u(s, y)=c_{1, \alpha}\left[(t+s)^{1-1 / \alpha}-|t-s|^{1-1 / \alpha}\right],
$$

where

$$
c_{1, \alpha}=\frac{1}{2 \pi(\alpha-1)} \Gamma\left(\frac{1}{\alpha}\right) .
$$

Consequently, it follows that the process $(u(t, x))_{t \geqslant 0}$ has the same law as the process $c_{2, \alpha} B^{1 / 2,1-1 / \alpha}$, where $B^{1 / 2,1-1 / \alpha}$ is a bi-fBm with Hurst parameters $H=$ $\frac{1}{2}$ and $K=1-1 / \alpha$ and

$$
c_{2, \alpha}^{2}=c_{1, \alpha} 2^{1-1 / \alpha}
$$


Pro of. We follow the lines of the proof of Proposition B.1. Assuming that $0 \leqslant s \leqslant t$, we have from (B.2)

$$
\begin{aligned}
\mathbf{E} u(t, x) u(s, x) & =\int_{0}^{t \wedge s} d u \int_{\mathbb{R}} d z G_{\alpha}(t-u, x-z) G_{\alpha}(s-u, x-z) \\
& =\int_{0}^{t \wedge s} d u \int_{\mathbb{R}} d \xi \mathcal{F} G_{\alpha}(t-u, \cdot)(\xi) \overline{\mathcal{F} G_{\alpha}(s-u, \cdot)(\xi)} \\
& =(\pi)^{-1} \int_{0}^{s} d u \int_{0}^{\infty} d \xi e^{-(t+s-2 u)|\xi|^{\alpha}} \\
& =(\pi)^{-1} 2 / \alpha \int_{0}^{\infty} d \xi|\xi|^{2 / \alpha-1} e^{-|\xi|^{2}} \int_{0}^{s} d u(t+s-2 u)^{-1 / \alpha}
\end{aligned}
$$

and the conclusion is obtained since

$$
\int_{0}^{\infty} d \xi|\xi|^{2 / \alpha-1} e^{-|\xi|^{2}}=\frac{1}{2} \Gamma\left(\frac{1}{\alpha}\right)
$$

Then

$\mathbf{E} u(t, x) u(s, x)=\frac{1}{2}(\pi)^{-1} \frac{2}{\alpha} \frac{1}{2} \Gamma\left(\frac{1}{\alpha}\right) \frac{1}{1-1 / \alpha}\left[(t+s)^{1-1 / \alpha}-|t-s|^{1-1 / \alpha}\right]$.

REMARK 3.1. If $\alpha=2$, then $c_{1, \alpha}=\frac{1}{2 \sqrt{\pi}}$ since $\Gamma\left(\frac{1}{2}\right)=\sqrt{\pi}$. We retrieve a well-known formula (see [21] or [23]).

From Proposition 3.2 we can deduce many properties of the process $t \rightarrow$ $u(t, x)$. In particular, for every $x \in \mathbb{R}$ :

- The process $(u(t, x))_{t \geqslant 0}$ is self-similar of order $\frac{1}{2}(1-1 / \alpha)$ and it is Hölder continuous of order $\delta$ for any $\delta \in\left(0, \frac{1}{2}(1-1 / \alpha)\right)$.

- We have the following decomposition in law: $u(t, x)+Y_{t}=C B_{t}^{\frac{1}{2}(1-1 / \alpha)}$, where $Y$ is a Gaussian process with absolute continuous paths and $B^{\frac{1}{2}(1-1 / \alpha)}$ denotes an fBm with Hurst index $\frac{1}{2}(1-1 / \alpha)$ and $C>0$ (see [14]).

Let us end this paragraph by stating the result on the behavior of the variation of the solution in time.

Proposition 3.3. Fix $A_{1}<A_{2}$ and $x \in \mathbb{R}$. Let $t_{j}=A_{1}+\frac{j}{n}\left(A_{2}-A_{1}\right)$, $n \geqslant 1, j=0,1, \ldots, n$, be a partition of the interval $\left[A_{1}, A_{2}\right]$. Then the process $(u(t, x), t \geqslant 0)$ admits variation of order $2 \alpha /(\alpha-1)$ which is equal to

$$
c_{2, \alpha}^{2 \alpha /(\alpha-1)} C_{1 / 2,1-1 / \alpha}\left(A_{2}-A_{1}\right)
$$

with $C_{1 / 2,1-1 / \alpha}\left(A_{2}-A_{1}\right)$ from (2.1) and $c_{2, \alpha}$ from (B.4).

Pro of. This is an immediate consequence of Propositions 2.$]$ and 3.2 . 
REMARK 3.2. For $\alpha=2$, we retrieve a result from [21]: the solution to the standard heat equation with time-space white noise has non-trivial quartic variation.

Concerning the renormalized $q$-variations, we have the following.

Proposition 3.4. Fix $A_{1}<A_{2}$ and $x \in \mathbb{R}$. Let $t_{j}=A_{1}+\frac{j}{n}\left(A_{2}-A_{1}\right)$, $n \geqslant 1, j=0,1, \ldots, n$, be a partition of the interval $\left[A_{1}, A_{2}\right]$. Then

$$
\begin{gathered}
\frac{1}{\sqrt{n}} \sum_{i=0}^{n-1}\left[\left(\frac{n^{(\alpha-1) /(2 \alpha)}}{c_{2, \alpha} 2^{1 /(2 \alpha)}\left(A_{2}-A_{1}\right)^{(\alpha-1) /(2 \alpha)}}\right)^{q}\left(u\left(t_{i+1}, x\right)-u\left(t_{i}, x\right)\right)^{q}-\mu_{q}\right] \\
\rightarrow N\left(0, \sigma_{\frac{1}{2}(1-1 / \alpha), q}^{2}\right)
\end{gathered}
$$

with $\sigma_{\frac{1}{2}(1-1 / \alpha), q}^{2}$ from (2.4).

Pro of. From [14] we know that

$$
\left(u(t, x)+C_{1} X_{t}\right)_{t \geqslant 0} \equiv^{(d)} c_{2, \alpha} 2^{1 /(2 \alpha)} B_{t}^{\frac{1}{2}(1-1 / \alpha)},
$$

where $\equiv^{(d)}$ means the equivalence of finite-dimensional distributions, $B^{\frac{1}{2}(1-1 / \alpha)}$ is an $\mathrm{fBm}$ with Hurst parameter $\frac{1}{2}(1-1 / \alpha), C_{1}>0$ and $\left(X_{t}\right)_{t \geqslant 0}$ is a Gaussian process which satisfies $\left([2.3)\right.$. Therefore, $\left(2^{-1 /(2 \alpha)} u(t, x), t \geqslant 0\right)$ is a perturbed $\mathrm{fBm}$ in the sense of Lemma 2.1 . Also note that its Hurst parameter is strictly less than $\frac{1}{2}$. We can then apply Lemma 2.$]$ to obtain the conclusion.

3.3. Behavior in space. An analysis of the process (3.3) with respect to its space variable has been done in [4]. Let us recall the main facts.

From Proposition 3.1 in [4] we know that for every $t>0$ the process $(u(t, x)$, $x \in \mathbb{R})$ can be decomposed as

$$
u(t, x) \equiv^{(d)} m_{\alpha} B^{(\alpha-1) / 2}(x)+S(x),
$$

where $B^{(\alpha-1) / 2}$ is a fractional Brownian motion with Hurst parameter $(\alpha-1) / 2 \in$ $\left[0, \frac{1}{2}\right],(S(x))_{x \in \mathbb{R}}$ is a centered Gaussian process with $C^{\infty}$ sample paths and $m_{\alpha}$ is the following numerical constant:

$$
m_{\alpha}=(2 \Gamma(\alpha)|\cos (\alpha \pi / 2)|)^{-1 / 2} .
$$

From the decomposition (3.5) we notice that the regularity of $x \rightarrow u(t, x)$ is given by the fractional Brownian motion $B^{(\alpha-1) / 2}$. In particular, for every $x, y \in \mathbb{R}$ and $t>0$

$$
\mathbf{E}|u(t, x)-u(t, y)|^{2} \leqslant C|x-y|^{\alpha-1},
$$


which implies that the function $x \rightarrow u(t, x)$ is Hölder continuous of order $\delta \in$ $(0,(\alpha-1) / 2)$. Actually, we have a more precise result in Lemma 2.1 in [4], i.e. for every $x, y \in \mathbb{R}$ and $t>0$ and for $\delta$ close to zero,

$$
\mathbf{E}|u(t, x+\delta)-u(t, x)|^{2}=m_{\alpha}^{2} \delta^{\alpha-1}+O\left(\delta^{2}\right) .
$$

From the decomposition (3.5) we can deduce the $q$-variation of the solution $u$ with respect to the space variable.

Proposition 3.5. Fix $A_{1}<A_{2}$ and $t>0$. Let $x_{j}=A_{1}+\frac{j}{n}\left(A_{2}-A_{1}\right)$ for $j=0, \ldots, n$ and $n \geqslant 1$. Then the process $(u(t, x), x \in \mathbb{R})$ has $2 /(\alpha-1)$ variation, i.e. we have the following limit in probability:

$$
\lim _{n \rightarrow \infty} \sum_{j=0}^{n-1}\left|u\left(t, x_{j+1}\right)-u\left(t, x_{j}\right)\right|^{2 /(\alpha-1)}=m_{\alpha}^{2 /(\alpha-1)} \mathbf{E}|Z|^{2 /(\alpha-1)}\left(A_{2}-A_{1}\right) .
$$

Proof. Notice that the process $S$ satisfies condition ([2.3)). This follows from [4], but also from the proof of Proposition 4.6 below. We can then apply Lemma 2.](1).

From Lemma 2.1 and (3.5) we have the following result.

Proposition 3.6. Fix $A_{1}<A_{2}$ and $t>0$. Let $x_{j}=A_{1}+\frac{j}{n}\left(A_{2}-A_{1}\right)$ for $j=0, \ldots, n$ and $n \geqslant 1$. Then, if $\alpha \in(1,2)$,

$$
\frac{1}{\sqrt{n}} \sum_{i=0}^{n-1}\left[\left(\frac{n^{(\alpha-1) / 2}}{m_{\alpha}}\right)^{q}\left(u\left(t, x_{i+1}\right)-u\left(t, x_{i}\right)\right)^{q}-\mu_{q}\right] \rightarrow N\left(0, \sigma_{(\alpha-1) / 2, q}^{2}\right) .
$$

If $\alpha=2\left(\right.$ i.e. $\left.(\alpha-1) / 2=\frac{1}{2}\right)$, then

$$
\frac{1}{\sqrt{n}} \sum_{i=0}^{n-1}\left[\frac{n^{\alpha-1}}{m_{2}^{2}}\left(u\left(t, x_{i+1}\right)-u\left(t, x_{i}\right)\right)^{2}-1\right] \rightarrow N\left(0, \sigma_{1 / 2,2}^{2}\right)
$$

with the constant $\sigma_{H, q}$ from (‥4).

P r o o f. It suffices to note that from [4] we obtain $\mathbf{E}|S(x)-S(y)|^{2} \leqslant c|t-s|^{2}$ and that the Hurst index $(\alpha-1) / 2$ is less than $\frac{1}{2}$.

Let us make some comments:

REMARK 3.3. (1) When $\alpha=2$, we have (exact) quadratic variation in space for the solution. We retrieve again a known result from [21].

(2) We notice that the regularity in space is $\alpha$ times the regularity in time (i.e. $(\alpha-1) /(2 \alpha)$-Hölder continuity in time and $(\alpha-1) / 2$-Hölder regularity in space). The phenomenon was known for $\alpha=2$. 


\section{HEAT EQUATION WITH FRACTIONAL LAPLACIAN AND WHITE-COLORED NOISE}

In this section, we will add a new paramter to the heat equation (B. D) by considering a Gaussian noise which behaves as a fractional Brownian motion in space, i.e. its spatial covariance is given by the so-called Riesz kernel. More precisely, we will consider the stochastic heat equation

$$
\frac{\partial}{\partial t} u(t, x)=-(-\Delta)^{\alpha / 2} u(t, x)+\dot{W}^{\gamma}(t, x), \quad t \geqslant 0, x \in \mathbb{R}^{d}
$$

with $u(0, x)=0$ for every $x \in \mathbb{R}^{d}$. In (4.J), $-(-\Delta)^{\alpha / 2}$ denotes the fractional Laplacian with exponent $\alpha / 2, \alpha \in(1,2]$, and $W^{\gamma}$ is the so-called white-colored noise, i.e. $W^{\gamma}(t, A), t \geqslant 0, A \in \mathcal{B}\left(\mathbb{R}^{d}\right)$, is a centered Gaussian field with covariance

$$
\mathbf{E} W^{\gamma}(t, A) W^{\gamma}(s, B)=(t \wedge s) \int_{A} \int_{B} f(x-y) d x d y,
$$

where $f$ is the so-called Riesz kernel of order $\gamma$ given by

$$
f(x)=R_{\gamma}(x):=g_{\gamma, d}\|x\|^{-d+\gamma}, \quad 0<\alpha<d,
$$

with $g_{\gamma, d}=2^{d-\gamma} \pi^{d / 2} \Gamma((d-\gamma) / 2) / \Gamma(\gamma / 2)$. In this case, if we consider the measure $\mu(d \xi)=\|\xi\|^{-\gamma} d \xi$, we have the identity

$$
\int_{\mathbb{R}^{d}} \int_{\mathbb{R}^{d}} \varphi(x) f(x-y) \psi(y) d x d y=(2 \pi)^{-d} \int_{\mathbb{R}^{d}} \mathcal{F} \varphi(\xi) \overline{\mathcal{F} \psi(\xi)} \mu(d \xi)
$$

for any $\varphi, \psi \in \mathcal{S}\left(\mathbb{R}^{d}\right)$ (the Schwartz space on $\mathbb{R}^{d}$ ).

As usual, the mild solution to (B.D) is given by

$$
u(t, x)=\int_{0}^{t} \int_{\mathbb{R}^{d}} G_{\alpha}(t-u, x-z) W^{\gamma}(d u, d z),
$$

where the integral $W^{\gamma}(d u, d z)$ is a Wiener integral with respect to the Gaussian noise $W^{\gamma}$.

Let us first give the necessary and sufficient condition for the existence of the mild solution.

PROPOSITION 4.1. The mild solution (4.4) to the heat equation (4.1) is welldefined if and only if

$$
d<\alpha+\gamma
$$

Moreover, in this case, for every $T>0$

$$
\sup _{t \in[0, T], x \in \mathbb{R}} \mathbf{E}|u(t, x)|^{2}<\infty .
$$


Pr o o f. Using the identity (4.3), we have for $t \geqslant 0, x \in \mathbb{R}^{d}$,

$$
\begin{aligned}
\mathbf{E} u(t, x)^{2} & =\int_{0}^{t} d u \int_{\mathbb{R}^{d}} \int_{\mathbb{R}^{d}} d y d z G_{\alpha}(t-u, x-z) G_{\alpha}(t-u, x-y)|y-z|^{-(d-\gamma)} \\
& =(2 \pi)^{-d} \int_{0}^{t} d u \int_{\mathbb{R}^{d}} d \xi e^{-2\|\xi\|^{\alpha} u}\|\xi\|^{-\gamma} \\
& =C_{1, \alpha, \gamma} \int_{0}^{t} d u u^{-(d-\gamma) / \alpha}
\end{aligned}
$$

with $C_{1, \alpha, \gamma}=(2 \pi)^{-d} \int_{\mathbb{R}^{d}} d \xi e^{-2\|\xi\|^{\alpha}}\|\xi\|^{-\gamma}<\infty$. The integral $d u$ is finite if and only if $1-(d-\gamma) / \alpha>0$, which implies (4.5). The last bound in the statement is also trivial by the above computation.

4.1. Behavior in time. In the next result we deduce the law of the Gaussian process $u(t, x), t \geqslant 0$, with $x \in \mathbb{R}^{d}$ fixed.

Proposition 4.2. For every $s, t \geqslant 0$ and for every $x \in \mathbb{R}^{d}$, we have

$$
\mathbf{E} u(t, x) u(s, x)=c_{1, \alpha, \gamma}\left[(t+s)^{1-(d-\gamma) / \alpha}-|t-s|^{1-(d-\gamma) / \alpha}\right],
$$

where

$$
c_{1, \alpha, \gamma}=(2 \pi)^{-d} \int_{\mathbb{R}^{d}} d \xi\|\xi\|^{-\gamma} e^{-\|\xi\|^{\alpha}} \frac{1}{2(1-(d-\gamma) / \alpha)} .
$$

Consequently, it follows that the process $(u(t, x))_{t \geqslant 0}$ has the same law as $c_{2, \alpha, \gamma}\left(B_{t}^{1 / 2,1-(d-\gamma) / \alpha}\right)_{t \geqslant 0}$, where $B^{1 / 2,1-(d-\gamma) / \alpha}$ is a bi-fBm with $H=\frac{1}{2}$ and $K=1-(d-\gamma) / \alpha$ and

$$
c_{2, \alpha, \gamma}^{2}=c_{1, \alpha, \gamma} 2^{1-(d-\gamma) / \alpha} .
$$

Pro of. As in the proof of Proposition 3.2 we have for $0 \leqslant s \leqslant t$ and for $x \in \mathbb{R}^{d}$,

$$
\begin{aligned}
\mathbf{E} u(t, x) u(s, x) & =(2 \pi)^{-d} \int_{0}^{t \wedge s} d u \int_{\mathbb{R}^{d}} d \xi\|\xi\|^{-\gamma} e^{-(t-u)\|\xi\|^{\alpha}} e^{-(s-u)\|\xi\|^{\alpha}} \\
& =(2 \pi)^{-d} \int_{\mathbb{R}^{d}} d \xi\|\xi\|^{-\gamma} e^{-\|\xi\|^{\alpha}} \int_{0}^{s} d u(t+s-2 u)^{-(d-\gamma) / \alpha} \\
& =c_{1, \alpha, \gamma}\left((t+s)^{1-(d-\gamma) / \alpha}-|t-s|^{1-(d-\gamma) / \alpha}\right)
\end{aligned}
$$

with $c_{1, \alpha, \gamma}$ given by (4.6). 
As a consequence, the process $t \rightarrow u(t, x)$ is Hölder continuous of order $\delta$ for every $\delta \in(0,1-(d-\gamma) / \alpha)$ and it is self-similar of order $\frac{1}{2}(1-(d-\gamma) / \alpha)$.

Now, it is immediate to obtain the $q$-variations of the process $u$ in time. The proof is similar to the proof of Proposition 3.3 .

Proposition 4.3. Fix $A_{1}<A_{2}$ and $x \in \mathbb{R}$. Let $t_{j}=A_{1}+\frac{j}{n}\left(A_{2}-A_{1}\right)$, $n \geqslant 1, j=0,1, \ldots, n$, be a partition of the interval $\left[A_{1}, A_{2}\right]$. Then the process $(u(t, x), t \geqslant 0)$ admits a variation of order $(2 \alpha) /(\alpha+\gamma-d)$ which is equal to

$$
c_{2, \alpha, \gamma}^{(2 \alpha) /(\alpha+\gamma-d)} C_{1 / 2,1-(d-\gamma) / \alpha}\left(A_{2}-A_{1}\right)
$$

with $C_{1 / 2,1-(d-\gamma) / \alpha}$ from (2.1) and $c_{2, \alpha, \gamma}$ from (4.7).

Moreover, from the proof of Proposition B.4, we have

Proposition 4.4. Fix $A_{1}<A_{2}$ and $x \in \mathbb{R}$. Let $t_{j}=A_{1}+\frac{j}{n}\left(A_{2}-A_{1}\right), n \geqslant 1$, $j=0,1, \ldots, n$, be a partition of the interval $\left[A_{1}, A_{2}\right]$. Then

$$
\begin{gathered}
\frac{1}{\sqrt{n}} \sum_{i=0}^{n-1}\left[\left(\frac{n^{(\alpha+\gamma-d) /(2 \alpha)}}{c_{2, \alpha, \gamma} 2^{(d-\gamma) /(2 \alpha)}\left(A_{2}-A_{1}\right)^{(\alpha+\gamma-d) /(2 \alpha)}}\right)^{q}\left(u\left(t_{i+1}, x\right)-u\left(t_{i}, x\right)\right)^{q}-\mu_{q}\right] \\
\rightarrow N\left(0, \sigma_{\frac{1}{2}(1-(d-\gamma) / \alpha), q}^{2}\right)
\end{gathered}
$$

with $\sigma_{\frac{1}{2}(1-1 / \alpha), q}^{2}$ from (2.4).

When $\gamma=0$ and $d=1$, we retrieve the result in the case of the white noise in space.

The next step is to study the behavior in space of (4.4). Now, we work in spatial dimension $d \geqslant 1$. In this case the solution will be related to the multiparameter isotropic fractional Brownian motion. For this reason, let us present below the definition and the basic properties of this process.

4.2. Isotropic fractional Brownian motion. In this paragraph we will use bold notation to indicate vectors in $\mathbb{R}^{d}$ to differentiate them from real numbers in order to avoid confusion.

The isotropic multiparameter $f B m$ (also known as the Lévy $f B m)\left(B^{H}(\mathbf{x}), \mathbf{x} \in\right.$ $\left.\mathbb{R}^{d}\right)$ with Hurst parameter $H \in(0,1)$ is defined as a centered Gaussian process, starting from zero, with covariance function

$$
\mathbf{E}\left(B^{H}(\mathbf{x}) B^{H}(\mathbf{y})\right)=\frac{1}{2}\left(\|\mathbf{x}\|^{2 H}+\|\mathbf{y}\|^{2 H}-\|\mathbf{x}-\mathbf{y}\|^{2 H}\right) \quad \text { for every } \mathbf{x}, \mathbf{y} \in \mathbb{R}^{d},
$$

where $\|\cdot\|$ denotes the Euclidean norm in $\mathbb{R}^{d}$. It can be also represented as a Wiener integral with respect to the Wiener sheet, see [8], [15].

The isotropic multiparameter $\mathrm{fBm}$ is self-similar and it has stationary increments in the following sense: 
for every $\mathbf{h} \in \mathbb{R}_{+}^{d}$

$$
\left(B^{H}(\mathbf{x}+\mathbf{h})-B^{H}(\mathbf{h})\right)_{\mathbf{x} \in \mathbb{R}_{+}^{d}} \equiv^{(d)}\left(B^{H}(\mathbf{x})\right)_{\mathbf{x} \in \mathbb{R}_{+}^{d}}
$$

and for every $a>0$

$$
\left(B^{H}(a \mathbf{x})\right)_{\mathbf{x} \in \mathbb{R}_{+}^{d}} \equiv^{(d)}\left(B^{H}(\mathbf{x})\right)_{\mathbf{x} \in \mathbb{R}_{+}^{d}} .
$$

Recall that we denoted by $\equiv^{(d)}$ the equivalence in the sense of finite-dimensional distributions. It is also possible to prove the stationarity of the increments in some generalized sense by using higher order difference (see Proposition 6 in [8]). An important property, which makes this Gaussian sheet different from the anisotropic fractional Brownian motion, is that for every $s, t \in \mathbb{R}^{d}$

$$
\mathbf{E}\left(B^{H}(\mathbf{x})-B^{H}(\mathbf{y})\right)^{2}=\|\mathbf{x}-\mathbf{y}\|^{2 H},
$$

which implies, due to the Gaussianity, that for every $n \geqslant 1$

$$
\mathbf{E}\left(B^{H}(\mathbf{x})-B^{H}(\mathbf{y})\right)^{n}=\mathbf{E}|Z|^{n}\|\mathbf{x}-\mathbf{y}\|^{n H},
$$

where $Z$ is a standard normal random variable. From (4.工) one can deduce, via a standard argument, the existence of a continuous version for $B^{H}$, see e.g. [113].

Following the one-parameter case, we define the $\alpha$-variation of the isotropic $\mathrm{fBm}$ as the limit in probability, as $n \rightarrow \infty$, of the sequence

$$
V_{\left[A_{1}, A_{2}\right]}^{n, q}\left(B^{H}\right)=\sum_{i=0}^{n-1}\left|B^{H}\left(\mathbf{x}_{i+1}\right)-B^{H}\left(\mathbf{x}_{i}\right)\right|^{q},
$$

where $\mathbf{x}_{i}=\left(x_{i}^{(1)}, \ldots, x_{i}^{(d)}\right)$ with $x_{i}^{(j)}=A_{1}+\frac{i}{n}\left(A_{2}-A_{1}\right)$ for $i=0, \ldots, n$ and $j=1, \ldots, d$.

Let us state the result on the variation of the isotropic fractional Brownian sheet. Even if its proof follows easily from the one-parameter case, it has not been stated before, as far as we know.

Proposition 4.5. The isotropic $f B m\left(\left(B^{H}(\mathbf{x}), \mathbf{x} \in \mathbb{R}^{d}\right)\right)$ has $1 / H$-variation over $\left[A_{1}, A_{2}\right]$ which is equal to

$$
\left(A_{2}-A_{1}\right) \mathbf{E}\left|B_{1}\right|^{1 / H}=\left(A_{2}-A_{1}\right) \sqrt{d} \mathbf{E}|Z|^{1 / H} .
$$

Pr o of. Consider the sequence

$$
Y_{n, q}=n^{q H-1} \sum_{i=0}^{n-1}\left|B^{H}\left(\mathbf{x}_{i+1}\right)-B^{H}\left(\mathbf{x}_{i}\right)\right|^{q} .
$$


By (4.10) and (4.9), it has the same law as

$$
Y_{n, q}^{\prime}=\left(A_{2}-A_{1}\right)^{q H} \frac{1}{n} \sum_{i=0}^{n-1}\left|B^{H}(\mathbf{j}+\mathbf{1})-B^{H}(\mathbf{j})\right|^{q}
$$

with $\mathbf{j}=(j, \ldots, j) \in \mathbb{R}^{d}$. The sequence $\left(B^{H}(\mathbf{j}+\mathbf{1})-B^{H}(\mathbf{j})\right)_{\mathbf{j} \in \mathbb{Z}^{d}}$ is stationary and has the same law as $d^{H / 2}\left(B_{j+1}-B_{j}\right)$, where $B$ is a one-parameter fBm with Hurst parameter $H$.

By the ergodic theorem, $Y_{n, q}^{\prime}$ converges to $\left(A_{2}-A_{1}\right)^{q H} \mathbf{E}\left|B_{1}\right|^{q}$. Taking $q=$ $1 / H$, we obtain the conclusion.

Following the proof of Lemma 2.1, we can get the $q$-variation of the isotropic $\mathrm{fBm}$ perturbed by a regular multiparameter process.

LEMMA 4.1. Let $\left(B^{H}(\mathbf{x})\right)_{\mathbf{x} \in \mathbb{R}^{d}}$ be a d-parameter isotropic fBm and consider $(X(x))_{x \in \mathbb{R}^{d}}$ a d-parameter stochastic process that satisfies

$$
\mathbf{E}|X(\mathbf{x})-X(\mathbf{y})|^{2} \leqslant C\|\mathbf{x}-\mathbf{y}\|^{2} \quad \text { for every } \mathbf{x}, \mathbf{y} \in \mathbb{R}^{d} .
$$

Define

$$
Y(\mathbf{x})=B^{H}(\mathbf{x})+X(\mathbf{x}) \quad \text { for every } \mathbf{x} \in \mathbb{R}^{d}
$$

Then:

(1) The process $(Y(\mathbf{x}))_{\mathbf{x} \in \mathbb{R}^{d}}$ has $1 / H$-variation which is equal to

$$
\left(A_{2}-A_{1}\right) \sqrt{d} \mathbf{E}|Z|^{1 / H} .
$$

(2) If $H \in\left(0, \frac{1}{2}\right)$ and $q \geqslant 2$, we have

$$
\begin{aligned}
\frac{1}{\sqrt{n}} V_{q, n}\left(Y^{H}\right)=\frac{1}{\sqrt{n}} \sum_{i=0}^{n-1}\left[\frac { n ^ { H q } d ^ { - H q / 2 } } { ( A _ { 2 } - A _ { 1 } ) ^ { q H } } \left(Y^{H}\left(\mathbf{x}_{i+1}\right)\right.\right. & \left.\left.-Y^{H}\left(\mathbf{x}_{i}\right)\right)^{q}-\mu_{q}\right] \\
& \rightarrow N\left(0, \sigma_{H, q}^{2}\right) .
\end{aligned}
$$

Pr o of. As in the proof of Lemma 2.1], we have the double inequality (2.6) due to the Minkowski inequality. The sequence

$$
\sum_{i=0}^{n-1}\left|B^{H}\left(\mathbf{x}_{i+1}\right)-B^{H}\left(\mathbf{x}_{i}\right)\right|^{1 / H}
$$

converges again almost surely and in $L^{1}$ to the desired limit, by Proposition 4.5. It remains to show that $\sum_{i=0}^{n-1}\left|X\left(\mathbf{x}_{i+1}\right)-X\left(\mathbf{x}_{i}\right)\right|^{1 / H}$ converges to zero in probability and this is an easy consequence of the assumption (4.12).

For (2), it suffices to observe that the vector $\left(B^{H}\left(\mathbf{x}_{i+1}\right)-B^{H}\left(\mathbf{x}_{i}\right)\right)_{0,1, \ldots, n-1}$ has the same law as $d^{H / 2}\left(B^{H}\left(x_{j+1}\right)-B\left(x_{j}\right)\right)_{0,1, n-1}$, where $B$ is a one-parameter fBm with Hurst parameter $H$, and to apply Lemma 2.1 . 
4.3. Behavior in space. We go back to the process (4.4) and analyze its behavior in space. We prove the following result.

PROPOSITION 4.6. Fix $t>0$. Then the process $(u(t, x))_{x \in \mathbb{R}^{d}}$ has the same finite-dimensional distribution as

$$
c_{3, \alpha, \gamma} B^{(\alpha+\gamma-d) / 2}(x)+S(x), \quad x \in \mathbb{R}^{d},
$$

where $B^{(\alpha+\gamma-d) / 2}$ stands for an isotropic multiparameter $f B m$ with Hurst index $(\alpha+\gamma-d) / 2, S(x), x \in \mathbb{R}^{d}$, is a Gaussian process with $C^{\infty}$ paths which satisfies (4.12) and

$$
c_{3, \alpha, \gamma}^{2}=(2 \pi)^{-d} \int_{\mathbb{R}^{d}} d w w^{-(\alpha+\gamma)}(1-\cos (w \cdot e)) .
$$

Proof. Define, for $x \in \mathbb{R}^{d}$,

$$
S(x)=\int_{t}^{\infty} \int_{\mathbb{R}^{d}}\left(G_{\alpha}(s, z)-G_{\alpha}(u, x-z)\right) W^{\gamma}(d s, d z) .
$$

Then we have

$$
\begin{aligned}
\mathbf{E}|S(x)|^{2}= & \int_{t}^{\infty} d s \int_{\mathbb{R}^{d}} \int_{\mathbb{R}^{d}} d z d z^{\prime}\left(G_{\alpha}(s, z)-G_{\alpha}(s, x-z)\right) \\
& \times\left(G_{\alpha}\left(s, z^{\prime}\right)-G_{\alpha}\left(s, x-z^{\prime}\right)\right) f\left(z-z^{\prime}\right) \\
= & (2 \pi)^{d} \int_{t}^{\infty} d u \int_{\mathbb{R}^{d}} d \xi\|\xi\|^{-\gamma}\left(\mathcal{F} G_{\alpha}(s, \cdot)(\xi)-\mathcal{F} G_{\alpha}(s, x-\cdot)(\xi)\right) \\
& \times \frac{\left(\mathcal{F} G_{\alpha}(s, \cdot)(\xi)-\mathcal{F} G_{\alpha}(s, x-\cdot)(\xi)\right)}{=} \\
= & (2 \pi)^{-d} \int_{t}^{\infty} d u \int_{\mathbb{R}^{d}} d \xi\|\xi\|^{-\gamma} e^{-2 u\|\xi\|^{\alpha}}\left|1-e^{-i \xi \cdot x}\right|^{2},
\end{aligned}
$$

where we used the Parseval formula (4.3). Now, by using Fubini's theorem and computing the integral $d u$, we get

$$
\mathbf{E}|S(x)|^{2}=(2 \pi)^{-d} \int_{\mathbb{R}^{d}} d \xi\|\xi\|^{-\gamma-\alpha}(1-\cos (\xi \cdot x)) e^{-2 t\|\xi\|^{\alpha}}<\infty .
$$

The function under the integral $d \xi$ is integrable at infinity because of the presence of the exponential function, while in the vicinity of zero we use $|1-\cos (\xi \cdot x)| \leqslant$ $c\|\xi\|^{2}$ and then

$$
\|\xi\|^{-\gamma-\alpha}(1-\cos (\xi \cdot x)) e^{-2 t\|\xi\|^{\alpha}} \leqslant C\|\xi\|^{-\alpha-\gamma+2},
$$

which is integrable for $\xi$ close to zero since $-\alpha-\gamma+2+d>0$. 
In the same way we can control the increments of $S$ to prove the relation (4.12). Indeed, for $x_{1}, x_{2} \in \mathbb{R}^{d}$,

$$
\begin{aligned}
\mathbf{E} \mid S\left(x_{2}\right) & -\left.S\left(x_{1}\right)\right|^{2}=(2 \pi)^{-d} \int_{t}^{\infty} d u \int_{\mathbb{R}^{d}} d \xi\|\xi\|^{-\gamma} e^{-2 u\|\xi\|^{\alpha}}\left|1-e^{-i \xi \cdot\left(x_{2}-x_{1}\right)}\right|^{2} \\
& =2(2 \pi)^{-d} \int_{t}^{\infty} d u \int_{\mathbb{R}^{d}} d \xi\|\xi\|^{-\gamma} e^{-2 u\|\xi\|^{\alpha}}\left(1-\cos \left(\xi \cdot\left(x_{2}-x_{1}\right)\right)\right) \\
& =(2 \pi)^{-d} \int_{\mathbb{R}^{d}} d \xi\|\xi\|^{-\gamma-\alpha}\left(1-\cos \left(\xi \cdot\left(x_{2}-x_{1}\right)\right)\right) e^{-2 t\|\xi\|^{\alpha}} \\
& \leqslant(2 \pi)^{d}\left\|x_{2}-x_{1}\right\|^{2} \int_{\mathbb{R}^{d}} d \xi\|\xi\|^{-\gamma-\alpha+2} e^{-2 t\|\xi\|^{\alpha}}
\end{aligned}
$$

since $1-\cos \left(\xi \cdot\left(x_{2}-x_{1}\right)\right) \leqslant\left\|x_{2}-x_{1}\right\|^{2}\|\xi\|^{2}$.

Put $U(x)=u(t, x)-S(x)$ for every $x \in \mathbb{R}^{d}$. We will show that $(U(x))_{x \in \mathbb{R}^{d}}$ is, modulo a constant, an isotropic $\mathrm{fBm}$. We can write, for $x, y \in \mathbb{R}$, by using the independence of $u$ and $S$ (because the noise $W^{\gamma}$ is white in time),

$$
\begin{aligned}
\mathbf{E}|U(x)-U(y)|^{2}= & \mathbf{E}\left[\int_{0}^{t} \int_{\mathbb{R}^{d}}\left(G_{\alpha}(u, x-z)-G_{\alpha}(u, y-z)\right) W^{\gamma}(d u, d z)\right]^{2} \\
& +\mathbf{E}\left[\int_{t}^{\infty} \int_{\mathbb{R}^{d}}\left(G_{\alpha}(u, x-z)-G_{\alpha}(u, y-z)\right) W^{\gamma}(d u, d z)\right]^{2} \\
& =\mathbf{E}\left[\int_{0}^{\infty} \int_{\mathbb{R}^{d}}\left(G_{\alpha}(u, x-z)-G_{\alpha}(u, y-z)\right) W^{\gamma}(d u, d z)\right]^{2} \\
& =2(2 \pi)^{-d} \int_{0}^{\infty} d u \int_{\mathbb{R}^{d}} d \xi\|\xi\|^{-\gamma} e^{-2 u\|\xi\|^{\alpha}}(1-\cos (\xi \cdot(x-y))) \\
& =(2 \pi)^{-d} \int_{\mathbb{R}^{d}} d \xi\|\xi\|^{-\gamma-\alpha}(1-\cos (\xi \cdot(x-y))),
\end{aligned}
$$

where we computed the integral $d u$. By making the change of variables $\xi\|y-x\|=$ $w$ and putting $e=(y-x) /\|y-x\|$, we have

$$
\mathbf{E}|U(x)-U(y)|^{2}=(2 \pi)^{-d}\|y-x\|^{\alpha+\gamma-d} \int_{\mathbb{R}^{d}} d w w^{-(\alpha+\gamma)}(1-\cos (w \cdot e)),
$$

which implies that $U$ coincides in law with $c_{3, \alpha, \gamma} B^{(\alpha+\gamma-d) / 2}$, where $B^{(\alpha+\gamma-d) / 2}$ is an isotropic $\mathrm{fBm}$ and $c_{3, \alpha, \gamma}^{2}$ given by (4.14).

By Lemma 4.] and Proposition 4.6, we have the following two propositions.

Proposition 4.7. The process $x \rightarrow u(t, x)$ has $2 /(\alpha+\gamma-d)$-variation given by $c_{3, \alpha, \gamma}^{2 /(\alpha+\gamma-d)}\left(A_{2}-A_{1}\right) \sqrt{d} \mathbf{E}|Z|^{2 /(\alpha+\gamma-d)}$. 
Proposition 4.8. Fix $A_{1}<A_{2}$ and $t>0$. Let $x_{j}^{k}=A_{1}+\frac{j}{n}\left(A_{2}-A_{1}\right)$ for $j=0, \ldots, n, n \geqslant 1$, and for every $k=1, \ldots, d$. Also, let $\mathbf{x}_{j}=\left(x_{j}^{1}, \ldots, x_{j}^{d}\right)$. Then, if $\alpha+\gamma-d<1$,

$$
\begin{array}{r}
\frac{1}{\sqrt{n}} \sum_{i=0}^{n-1}\left[\left(\frac{n^{(\alpha+\gamma-d) / 2} d^{-H / 2}}{c_{3, \alpha, \gamma}}\right)^{q}\left(u\left(t, \mathbf{x}_{i+1}\right)-u\left(t, \mathbf{x}_{i}\right)\right)^{q}-\mu_{q}\right] \\
\rightarrow N\left(0, \sigma_{(\alpha+\gamma-d) / 2, q}^{2}\right)
\end{array}
$$

with $c_{3, \alpha, \gamma}$ from (4.14) and $\sigma_{(\alpha+\gamma-d) / 2, q}$ from (B.6).

Notice that we restricted ourselves to the situation $\alpha+\gamma-d<1$, which means that the parameter of the isotropic $\mathrm{fBm}$ associated with the solution is strictly less than one half, and we can apply Lemma 4.1.

\section{REFERENCES}

[1] P. Biler and W. A. Woyczyński, Global and exploding solutions for nonlocal quadratic evolution problems, SIAM J. Appl. Math. 59 (3) (1999), pp. 845-869.

[2] P. Breuer and P. Major, Central limit theorems for nonlinear functionals of Gaussian fields, J. Multivariate Anal. 13 (3) (1983), pp. 425-441.

[3] R. L. Dobrushin and P. Major, Non-central limit theorems for nonlinear functionals of Gaussian fields, Z. Wahrsch. Verw. Gebiete 50 (1) (1979), pp. 27-52.

[4] M. Foondun, D. Khoshnevisan, and P. Mahboubi, Analysis of the gradient of the solution to a stochastic heat equation via fractional Brownian motion, Stoch. Partial Differ. Equ. Anal. Comput. 3 (2) (2015), pp. 133-158.

[5] M. Foondun, J. B. Mijena, and E. Nane, Non-linear noise excitation for some spacetime fractional stochastic equations in bounded domains, Fract. Calc. Appl. Anal. 19 (6) (2016), pp. 1527-1553.

[6] L. Giraitis and D. Surgailis, A central limit theorem for quadratic forms in strongly dependent linear variables and its application to asymptotical normality of Whittle's estimate, Probab. Theory Related Fields 86 (1) (1990), pp. 87-104.

[7] D. Harnett and D. Nualart, Decomposition and limit theorems for a class of self-similar Gaussian processes, in: Stochastic Analysis and Related Topics: A Festschrift in Honor of Rodrigo Bañuelos, F. Baudoin and J. Peterson (Eds.), Springer International Publishing, Cham 2017, pp. 99-116.

[8] E. Herbin, From $N$ parameter fractional Brownian motions to $N$ parameter multifractional Brownian motions, Rocky Mountain J. Math. 36 (4) (2006), pp. 1249-1284.

[9] C. Houdré and J. Villa, An example of infinite dimensional quasi-helix, in: Stochastic Models, Contemp. Math. 336 (2003), pp. 195-201.

[10] N. Jacob and H. Leopold, Pseudo-differential operators with variable order of differentiation generating Feller semigroups, Integral Equations Operator Theory 17 (4) (1993), pp. 544-553.

[11] N. Jacob, A. Potrykus, and J. Wu, Solving a non-linear stochastic pseudo-differential equation of Burgers type, Stochastic Process. Appl. 120 (12) (2010), pp. 2447-2467.

[12] Y. Jiang, K. Shi, and Y. Wang, Stochastic fractional Anderson models with fractional noises, Chin. Ann. Math. Ser. B 31 (1) (2010), pp. 101-118.

[13] D. Khoshnevisan, Multiparameter Processes: An Introduction to Random Fields, Springer, New York 2002.

[14] P. Lei and D. Nualart, A decomposition of the bifractional Brownian motion and some applications, Statist. Probab. Lett. 79 (5) (2009), pp. 619-624. 
[15] T. Lindstrøm, Fractional Brownian fields as integrals of white noise, Bull. Lond. Math. Soc. 25 (1) (1993), pp. 83-88.

[16] W. Liu, K. Tian, and M. Foondun, On some properties of a class of fractional stochastic heat equations, J. Theoret. Probab. 30 (4) (2017), pp. 1310-1333.

[17] M. M. Meerschaert, E. Nane, and Y. Xiao, Fractal dimension results for continuous time random walks, Statist. Probab. Lett. 83 (4) (2013), pp. 1083-1093.

[18] I. Nourdin, D. Nualart, and C. A. Tudor, Central and non-central limit theorems for weighted power variations of fractional Brownian motion, Ann. Inst. Henri Poincaré Probab. Stat. 46 (4) (2010), pp. 1055-1079.

[19] D. Nualart, The Malliavin Calculus and Related Topics, second edition, Springer, Berlin 2006.

[20] J. Pospisil and R. Tribe, Parameter estimates and exact variations for stochastic heat equations driven by space-time white noise, Stoch. Anal. Appl. 25 (3) (2007), pp. 593-611.

[21] J. Swanson, Variations of the solution to a stochastic heat equation, Ann. Probab. 35 (6) (2007), pp. 2122-2159.

[22] A. Truman and $\mathrm{J}$. Wu, On a stochastic nonlinear equation arising from $1 D$ integrodifferential scalar conservation laws, J. Funct. Anal. 238 (2) (2006), pp. 612-635.

[23] C. A. Tudor, Analysis of Variations for Self-similar Processes: A Stochastic Calculus Approach, Springer, Cham 2013.

[24] C. A. Tudor and Y. Xiao, Sample paths of the solution to the fractional-colored stochastic heat equation, Stoch. Dyn. 17 (1) (2017), 1750004.

Z. Mahdi Khalil

Laboratoire Paul Painlevé

CNRS UMR 8524

Université de Lille

59655 Villeneuve d'Ascq

France

E-mail: zeina-kh@outlook.fr
C. A. Tudor

Laboratoire Paul Painlevé

CNRS UMR 8524

Université de Lille 59655 Villeneuve d'Ascq

France

E-mail: tudor@math.univ-lille1.fr

Received on 21.11.2017;

revised version on 5.4 .2018 